\title{
Investigation of Hemp (Cannabis sativa L.) Fibre Quantity and Quality as Influenced by Genotype and Seed Rate
}

\author{
Zofija Jankauskienė ${ }^{1}$, Elvyra Gruzdevienė ${ }^{2}$, Ernestas Maumevičius ${ }^{3}$, Iryna M. Layko ${ }^{4}$ \\ ${ }^{1,2}$ Upyte Experimental Station of the Lithuanian Research Centre for Agriculture and Forestry \\ Address: Linininku 3, Upytè, Panevėžys district, LT-38 294, Lithuania \\ ${ }^{3}$ Aleksandras Stulginskis University \\ Address: Studentu 11, Akademija, Kaunas district, LT-53067, Lithuania \\ ${ }^{4}$ Research Station of Bast Crops of IA Northern East NAAS \\ Address: 45 Tereshchenkiv Street, 41400 Hlukhiv, Sumy region, Ukraine
}

\begin{abstract}
The investigation on hemp fibre quantity and quality as influenced by genotype and seed rate was carried out at the Upyte Experimental Station Lithuanian of the Lithuanian Research Centre for Agriculture and Forestry in 2014. Bi-factorial trial was carried out: Factor A - variety (A1 - USO 31; A2 - Bialobrzeskie); Factor B - sowing rate (B1 - 45 $\mathrm{kg} \mathrm{ha}{ }^{-1} ; B 2-70 \mathrm{~kg} \mathrm{ha}^{-1}$ ). Data showed that variety (genotype) had a significant influence on fibre content (after dewretting as well after water-retting) - it was higher for variety Bialobrzeskie and amounted to 37.2 and $34.5 \%$, respectively. Seed rate (or crop density) did not show significant influence neither on fibre content nor on fibre quality (flexibility, strength) parameters.
\end{abstract}

Keywords: Cannabis sativa L., density, fibre, quality, seed rate, variety.

\section{INTRODUCTION}

The interest in hemp (Cannabis sativa L.) as an industrial plant, its numerous application ways, as well as in ecology of it, is growing up continuously. In recent five years only in Lithuania the area of industrial hemp increased as much as forty times from 54 ha in 2011, to 2345 ha in 2015 and 2454 ha in 2016 [1].

The yielding capacities of hemp and fibre quality depend on many factors such as genotype, seed rate, growing technology, pedoclimatic conditions, etc. [2; $3 ; 4]$.

Dew-retting and water-retting are two retting methods allowing fibre extraction from the stems of bast fibre plants, and both of them produce fibres of different quality, thus both ways should be investigated [3].

Different range of seed rates for hemp is reported when growing it for different purposes. Recommended sowing rates for fibre hemp vary between 40 and $150 \mathrm{~kg}$ per ha [5]. For textile purposes suggested seed rate varies from 40 to $80 \mathrm{~kg}$ ha $^{-1}[2 ; 6 ; 7 ; 8 ; 9 ; 10]$.

The goal of the investigation was to evaluate how the hemp variety (genotype) and sowing rate influences on fibre quantity and quality indices.

\section{MATERIALS AND METHODS}

The trial was conducted at the Upyte Experimental Station of the Lithuanian Research Centre for Agriculture and Forestry in 2014. The soil - an Eutri-Endohypogleyic Cambisol, CMg-n-weu [11]. The $\mathrm{pH}_{\mathrm{KC}} \mathrm{l}$ level was 6.8 (potentiometrically), humus concentration $-2.23 \%$ (by Hereus apparatus), content of available phosphorus $\left(\mathrm{P}_{2} \mathrm{O}_{5}\right)$ in the soil plough layer was $116 \mathrm{mg} \mathrm{kg}^{-1}$, the content of available potassium $\left(\mathrm{K}_{2} \mathrm{O}\right)-85 \mathrm{mg} \mathrm{kg}^{-1}$ (determined in A-L extraction). Hemp followed winter wheat in the field rotation. Before sowing, complex fertilizers N9-P25-K25 (200 kg ha-1) have been applied.

Bi-factorial trial was carried out: Factor A variety (A1 - USO 31; A2 - Bialobrzeskie); Factor $\mathrm{B}$ - sowing rate $\left(\mathrm{B} 1\right.$ - $45 \mathrm{~kg} \mathrm{ha}^{-1}$; $\mathrm{B} 2$ - $\left.70 \mathrm{~kg} \mathrm{ha}^{-1}\right)$. Both of selected varieties are monoecious.

Hemp was sown on $7^{\text {th }}$ of May in $15 \mathrm{~cm}$ inter-row spacing by single-row sowing machine. The size of trial plots was $2 \times 5=10 \mathrm{~m}^{2}$, the size of record plots $-2 \times 4=8 \mathrm{~m}^{2}$ (trial was sown in tree replications). Randomised plot design was used. At both sides of the trial the protective plots of the same size as record plots were sown.

Hemp crop density was assessed at hemp harvest time. For this purpose 4 microplots of $0.25 \times 0.25 \mathrm{~m}$ were marked in each trial plot after full crop emergence. Hemp was harvested when the first matured seed appeared (26 $6^{\text {th }}$ of August). http://dx.doi.org/ 10.17770/etr2017voll.2530 
After morphological analyses, the index, important for fibre quantity and quality, was calculated as the ratio between technical stem length and stem diameter $[4 ; 12]$.

Before starting dew or water-retting, technical stalk part from hemp stalks was prepared (cutting away the top part of plant containing panicle and still left on the stem branches with leaves). One part of hemp stalk samples $(0.5 \mathrm{~kg}$ per plot $)$ was water-retted (temperature $\left.37^{\circ} \mathrm{C}\right)$ for 5 days, other part $(0.5 \mathrm{~kg}$ per plot) was dew-retted on the grassland for 2 weeks; then dry straw was weighed and beaked by laboratory tool LM-3; obtained material was shaked by hand until the shives were withdrawn. Obtained fiber was weighed and fiber content in the straw was calculated by the formula (1):

$$
\mathrm{Fc}=\mathrm{Wf} \times 100 / \text { Wstr }
$$

where: Fc - fibre content in the straw, \%,

Wf - weight of obtained fibre, $g$,

Wstr - weight of straw before scutching, g.

Later on fiber content in the stalks was recounted (2):

$\mathrm{Fc}=\mathrm{Wf} \times 100 / \mathrm{Ws}$

where: Fc - fibre content in the stalks, \%,

Wf - weight of obtained fibre, $\mathrm{g}$,

Ws - weight of stalks before retting, $g$.

Fibre flexibility was evaluated by a device G-2, strength of fibre - by a device DK-60 [13].

For statistical data evaluation the statistical software developed in the Lithuanian Institute of Agriculture was used, ANOVA method applied [14].

Mean air temperature and amount of precipitation were assessed during hemp growing period (Table 1).

Table 1.

Mean weather temperature and precipitation during hemp growing period

Upytè, 2014

\begin{tabular}{|c|l|c|c|c|c|}
\hline \multirow{4}{*}{ Month } & \multirow{2}{*}{$\begin{array}{c}\text { Ten-day } \\
\text { period }\end{array}$} & \multicolumn{2}{|c|}{$\begin{array}{c}\text { Mean weather } \\
\text { temperature, }\end{array}$} & \multicolumn{2}{c|}{ Rainfall, mm } \\
\cline { 3 - 6 } & 2014 & $\begin{array}{c}\text { Long- } \\
\text { term } \\
\text { average }\end{array}$ & 2014 & $\begin{array}{c}\text { Long- } \\
\text { term } \\
\text { average }\end{array}$ \\
\hline \multirow{4}{*}{ May } & I & 8.2 & 11.0 & 23.5 & 16.0 \\
\cline { 2 - 6 } & II & 13.8 & 12.6 & 34.0 & 16.0 \\
\cline { 2 - 6 } & III & 16.8 & 13.5 & 7.5 & 18.0 \\
\cline { 2 - 6 } & Aver./total & $\mathbf{1 2 . 9}$ & $\mathbf{1 2 . 4}$ & $\mathbf{6 5 . 0}$ & $\mathbf{5 0 . 0}$ \\
\hline \multirow{5}{*}{ June } & I & 17.0 & 14.4 & 12.0 & 22.0 \\
\cline { 2 - 6 } & II & 13.2 & 15.3 & 27.0 & 23.0 \\
\cline { 2 - 6 } & III & 12.6 & 16.2 & 71.0 & 24.0 \\
\cline { 2 - 6 } & Aver./total & $\mathbf{1 4 . 3}$ & $\mathbf{1 5 . 3}$ & $\mathbf{1 1 0 . 0}$ & $\mathbf{6 9 . 0}$ \\
\hline \multirow{5}{*}{ July } & I & 19.0 & 17.2 & 49.5 & 25.0 \\
\cline { 2 - 6 } & II & 18.8 & 18.0 & 20.0 & 25.0 \\
\cline { 2 - 6 } & III & 21.9 & 18.0 & 23.0 & 26.0 \\
\cline { 2 - 6 } & Aver./total & $\mathbf{1 9 . 9}$ & $\mathbf{1 7 . 7}$ & $\mathbf{9 2 . 5}$ & $\mathbf{7 6 . 0}$ \\
\hline August & I & 22.4 & 17.2 & 58.0 & 28.0 \\
\cline { 2 - 6 } & II & 17.0 & 16.1 & 35.5 & 29.0 \\
\cline { 2 - 6 } & III & 13.2 & 15.0 & 79.5 & 28.0 \\
\cline { 2 - 6 } & Aver./total & $\mathbf{1 7 . 4}$ & $\mathbf{1 6 . 1}$ & $\mathbf{1 7 3 . 0}$ & $\mathbf{8 5 . 0}$ \\
\hline
\end{tabular}

Hemp germinated approximately in two weeks after sowing. It was warm and rainy in the middle of May (the amount of precipitation was twice more than the long-term average for second ten-day period of May). June was slightly cooler that long-term average but abundant in precipitation. It was warm in July and August, but the amount of precipitation was again huge, and even oversupply as the water for some time was flooding some surfaces on the trial field. Hemp was thriving over the vegetation period.

\section{RESULTS AND DISCUSSION}

No doubts, that seed rate (Factor $B$ ) could have influence on crop density. The data of investigation show that seed rate had a significant influence on crop density at hemp harvest time (Table 2). The mean crop density was close 118 plants $\mathrm{m}^{-2}$ at seed rate of $45 \mathrm{~kg} \mathrm{ha}^{-1}$, and significantly higher -188 plants $\mathrm{m}^{-2}-$ at seed rate of $70 \mathrm{~kg} \mathrm{ha}^{-1}$. Also some significant interaction of tested factors was found crop density was significantly higher when sowing hemp of both varieties at seed rate of $70 \mathrm{~kg} \mathrm{ha}^{-1}$, than that when sowing at $45 \mathrm{~kg} \mathrm{ha}^{-1}$.

Table 2 .

Crop density (plants m-2) at hemp at harvest Upytè, 2014

\begin{tabular}{|c|c|c|c|}
\hline \multirow{2}{*}{$\begin{array}{c}\text { Variety } \\
\text { (Factor A) }\end{array}$} & \multicolumn{2}{|c|}{ Seed rate (Factor B) } & $\begin{array}{c}\text { Mean for } \\
\text { Factor A }\end{array}$ \\
\cline { 2 - 3 } & $45 \mathrm{~kg} \mathrm{ha}^{-1}$ & $70 \mathrm{~kg} \mathrm{ha}^{-1}$ & 149.8 \\
\hline USO 31 & 104.7 & $195.0^{*}$ & 155.0 \\
\hline Bialobrzeskie & 130.3 & $179.7^{*}$ & - \\
\hline Mean for Factor B & $117.5^{*}$ & $187.3^{*}$ & (variety x seed \\
\hline $\mathrm{R}_{05}$ (variety) $=17.23$ & $\begin{array}{c}\mathrm{R}_{05} \text { (seed rate) }=17.23 \mathrm{R}_{05} \\
\text { rate) }=29.85\end{array}$ \\
\hline
\end{tabular}

As fibre content and quality could be influenced by many factors, thus the additional index, conventionally related to fibre quantity and quality, was calculated. Relational index of distribution of fibres in the stem is the ratio between the technical stem length and the stem diameter - the higher is the ratio, the better is the fibre quality $[4 ; 12 ; 15]$. The results of our investigation show that this index was significantly influenced by seed rate [16]. The plants sown at higher seed rate $\left(70 \mathrm{~kg} \mathrm{ha}^{-1}\right)$ had a higher ratio between the technical stem length and the stem diameter (376.8) than that sown at lower seed rate (45 $\left.\mathrm{kg} \mathrm{ha}^{-1}\right)$ (319.7) (Table 3).

Table 3.

The ratio between technical hemp stem length and stem diameter Upytè, 2014

\begin{tabular}{|c|c|c|c|}
\hline \multirow{2}{*}{$\begin{array}{c}\text { Variety } \\
\text { (Factor A) }\end{array}$} & \multicolumn{2}{|c|}{ Seed rate (Factor B) } & \multirow{2}{*}{$\begin{array}{l}\text { Mean for } \\
\text { Factor A }\end{array}$} \\
\hline & $45 \mathrm{~kg} \mathrm{ha}^{-1}$ & $70 \mathrm{~kg} \mathrm{ha}^{-1}$ & \\
\hline USO 31 & 303.8 & $386.4^{*}$ & 345.1 \\
\hline Bialobrzeskie & 335.6 & $367.3^{*}$ & 351.4 \\
\hline Mean for Factor B & $319.7 *$ & $376.8 *$ & - \\
\hline \multicolumn{4}{|c|}{$\begin{array}{cc}\mathrm{R}_{05} \text { (variety) }=21.86 & \mathrm{R}_{05}(\text { seed rate })=21.86 \mathrm{R}_{05} \text { (variety x seed } \\
\text { rate })=37.86\end{array}$} \\
\hline
\end{tabular}


Environment. Technology. Resources, Rezekne, Latvia Proceedings of the $11^{\text {th }}$ International Scientific and Practical Conference. Volume I, 125-128

Hemp genotype (variety), as Factor A, showed a significant influence on the fibre content after dewretting (Table 4). Variety Bialobrzeskie had significantly higher fibre content after dew-retting $(37.2 \%)$ than that of variety USO 31 (31.2\%). Unfortunately, the seed rate did not show any influence to this tested parameter.

The results of hemp fibre quality showed that neither fibre flexibility nor fibre strength was influenced by tested Factors (Tables 5 and 6). Hemp fibre after dew-retting was rough, inflexible, but enough firm.

Table 4.

Fibre content $(\%)$ in dew-retted stalks Upytè, 2014

\begin{tabular}{|c|c|c|c|}
\hline \multirow{2}{*}{$\begin{array}{c}\text { Variety } \\
\text { (Factor A) }\end{array}$} & \multicolumn{2}{|c|}{ Seed rate (Factor $\mathrm{B})$} & \multirow{2}{*}{$\begin{array}{l}\text { Mean for } \\
\text { Factor A }\end{array}$} \\
\hline & $45 \mathrm{~kg} \mathrm{ha}^{-1}$ & $70 \mathrm{~kg} \mathrm{ha}^{-1}$ & \\
\hline USO 31 & 31.3 & 31.0 & $31.2 *$ \\
\hline Bialobrzeskie & 37.7 & 36.7 & $37.2 *$ \\
\hline Mean for Factor B & 34.5 & 33.8 & - \\
\hline $\mathrm{R}_{05}$ (variety) $=0.88$ & \multicolumn{3}{|c|}{$\begin{array}{c}\mathrm{R}_{05}(\text { seed rate })=0.88 \mathrm{R}_{05}(\text { variety } x \text { seed rate }) \\
=1.53\end{array}$} \\
\hline
\end{tabular}

Table 5.

Dew-retted hemp fibre flexibility (mm) Upytè, 2014

\begin{tabular}{|c|c|c|c|}
\hline \multicolumn{4}{|c|}{ (Pyou, } \\
\hline \multirow{2}{*}{$\begin{array}{c}\text { Variety } \\
\text { (Factor A) }\end{array}$} & \multicolumn{2}{|c|}{ Seed rate (Factor B) } & \multirow{2}{*}{$\begin{array}{l}\text { Mean for } \\
\text { Factor A }\end{array}$} \\
\hline & $45 \mathrm{~kg} \mathrm{ha}^{-1}$ & $70 \mathrm{~kg} \mathrm{ha}^{-1}$ & \\
\hline USO 31 & 16.8 & 19.6 & 18.2 \\
\hline Bialobrzeskie & 17.3 & 18.7 & 18.0 \\
\hline Mean for Factor B & 17.1 & 19.1 & - \\
\hline $\mathrm{R}_{05}$ (variety) $=1.10$ & \multicolumn{3}{|c|}{$\begin{array}{l}\mathrm{R}_{05}(\text { seed rate })=1.10 \quad \mathrm{R}_{05} \text { (variety } \times \text { seed } \\
\text { rate })=1.90\end{array}$} \\
\hline
\end{tabular}

Table 6.

Dew-retted hemp fibre strength $(\mathrm{kg} \mathrm{F})$ Upytè, 2014

\begin{tabular}{|c|c|c|c|}
\hline $\begin{array}{c}\text { Variety } \\
\text { (Factor A) }\end{array}$ & \multicolumn{2}{|c|}{ Seed rate (Factor B) } & Mean for \\
\cline { 2 - 3 } & $45 \mathrm{~kg} \mathrm{ha}^{-1}$ & $70 \mathrm{~kg} \mathrm{ha}^{-1}$ & Factor A \\
\hline USO 31 & 19.8 & 17.8 & 18.8 \\
\hline Bialobrzeskie & 17.9 & 18.7 & 18.3 \\
\hline Mean for Factor B & 18.8 & 18.2 & - \\
\hline \multicolumn{3}{|c|}{$\mathrm{R}_{05}$ (variety) $=2.26$} & $\begin{array}{c}\mathrm{R}_{05} \text { (seed rate) }=2.26 \quad \mathrm{R}_{05} \text { (variety x seed } \\
\text { rate) }=3.92\end{array}$ \\
\hline
\end{tabular}

Fibre content in hemp stems was slightly higher after dew-retting then that after water-retting. It means that more fibre could be obtained applying dew-retting method. Nevertheless, fibre quality parameters also should be taken into account.

The influence of hemp genotype (variety), as Factor A, again was highlighted on fibre content after water-retting (Table 7). Variety Bialobrzeskie had significantly higher fibre content after water-retting $(34.5 \%)$ than that of variety USO 31 (29.2\%). Unfortunately, the seed rate did not show any significant influence again.

The fibre flexibility after water-retting was more than twice higher than that after dew-retting (Table 8). Such high value of flexibility exceeded some results of previous trials [3], and sometimes could be related to flax fibres [17].

Only some tendencies could be noted that fibre flexibility was slightly higher when hemp grew at higher plant density, but the differences are insignificant.

Table 7.

Fibre content (\%) in water-retted stalks Upyte, 2014

\begin{tabular}{|c|c|c|c|}
\hline \multirow{2}{*}{$\begin{array}{c}\text { Variety } \\
\text { (Factor A) }\end{array}$} & \multicolumn{2}{|c|}{ Seed rate (Factor B) } & Mean for \\
\hline & $45 \mathrm{~kg} \mathrm{ha}^{-1}$ & $70 \mathrm{~kg} \mathrm{ha}^{-1}$ & Factor A \\
\hline USO 31 & 29.2 & 29.1 & $29.1 *$ \\
\hline Bialobrzeskie & 34.3 & 34.7 & $34.5^{*}$ \\
\hline Mean for Factor B & 31.8 & 31.9 & - \\
\hline $\mathrm{R}_{05}$ (variety) $=1.22$ & \multicolumn{3}{|c|}{$\begin{array}{c}\mathrm{R}_{05} \text { (seed rate) }=1.22 \mathrm{R}_{05} \text { (variety } \mathrm{x} \text { seed rate) } \\
=1.395\end{array}$} \\
\hline
\end{tabular}

Table 8 .

Water-retted hemp fibre flexibility $(\mathrm{mm})$ Upytè, 2014

\begin{tabular}{|c|c|c|c|}
\hline \multirow{2}{*}{$\begin{array}{c}\text { Variety } \\
\text { (Factor A) }\end{array}$} & \multicolumn{2}{|c|}{ Seed rate (Factor B) } & \multirow{2}{*}{$\begin{array}{c}\text { Mean for } \\
\text { Factor A }\end{array}$} \\
\cline { 2 - 3 } & $45 \mathrm{~kg} \mathrm{ha}^{-1}$ & $70 \mathrm{~kg} \mathrm{ha}^{-1}$ & 47.7 \\
\hline USO 31 & 44.6 & 50.9 & 47.0 \\
\hline Bialobrzeskie & 46.1 & 47.8 & - \\
\hline Mean for Factor B & 45.4 & 49.4 & $\mathrm{R}_{05}$ (variety x seed \\
\hline \multicolumn{2}{|c|}{$\begin{array}{c}\mathrm{R}_{05} \text { (variety) }=1.65 \\
\text { rate) }=4.34\end{array}$} \\
\hline
\end{tabular}

In 2014, the fibre strength after dew-retting and water-retting was quite similar (Tables 6 and 9), and was much higher than in previous trials [3].

The differences in fibre strength between treatments were not established. Neither seed rate nor variety did not show any significant influence on fibre strength after water-retting.

Table 9.

Water-retted hemp fibre strength (kg F) Upytè, 2014

\begin{tabular}{|c|c|c|c|}
\hline \multirow{2}{*}{$\begin{array}{c}\text { Variety } \\
\text { (Factor A) }\end{array}$} & \multicolumn{2}{|c|}{ Seed rate (Factor B) } & \multirow{2}{*}{$\begin{array}{l}\text { Mean for } \\
\text { Factor A }\end{array}$} \\
\hline & $45 \mathrm{~kg} \mathrm{ha}^{-1}$ & $70 \mathrm{~kg} \mathrm{ha}^{-1}$ & \\
\hline USO 31 & 17.9 & 19.0 & 18.4 \\
\hline Bialobrzeskie & 19.5 & 19.8 & 19.6 \\
\hline Mean for Factor B & 18.7 & 19.4 & - \\
\hline \multicolumn{4}{|c|}{$\begin{array}{ccc}\mathrm{R}_{05} \text { (variety) }=1.46 & \mathrm{R}_{05} \text { (seed rate) }=1.46 \mathrm{R}_{05} \text { (variety x seed } \\
& \text { rate })=2.54\end{array}$} \\
\hline
\end{tabular}

Nevertheless the hemp plants sown at higher seed rate $\left(70 \mathrm{~kg} \mathrm{ha}^{-1}\right)$ had a higher ratio between the technical stem length and the stem diameter than that sown at lower seed rate $\left(45 \mathrm{~kg} \mathrm{ha}^{-1}\right)$ this was not reflected on fibre content or quality.

\section{CONCLUSION}

Seed rate had a significant influence on crop density at harvesting time. Variety (genotype) had a significant influence on fibre content (after dewretting as well after water-retting) - it was higher for variety Bialobrzeskie, and amounted to 37.2 and $34.5 \%$, respectively. Seed rate (or crop density) did not show significant influence neither on fibre content nor on fibre quality (flexibility, strength) parameters.

\section{REFERENCES}

[1] V. Jonaitienè, Z. Jankauskienè, and I. Stuogè, "Hemp Cultivation Opportunities and Perspectives in Lithuania", in Natural Fibres: Advances in Science and Technology Towards Industrial Applications From Science to Market 
(Eds: Raul Fangueiro, Sohel Rana), RILEM Bookseries, Vol. 12, 2016, pp. 407-414.

[2] S. Amaducci and H.-J. Gusovius, "Hemp - cultivation, extraction and processing", in Industrial Application of Natural Fibres: Properties and Technical Application (Ed. Müssing J.), A JohnWiley \& Sons, UK, 2010, pp. 109-134.

[3] Z. Jankauskienè and E. Gruzdevienè, "Physical parameters of dew retted and water retted hemp (Cannabis sativa L.) fibers", Zemdirbyste-Agriculture, 100 (1), 2013, pp. 71-80.

[4] М.Д. Мигаль, Біологія луб'яних волокон конопель. Суми: ТОВ „ТД Папірус“, 2011, 390 c.

[5] H. M. G. Van der Werf, W. C. A. van Geel and M. Wijlhuizen, "Agronomic research on hemp (Cannabis sativa L.) in the Netherlands, 1987-1993". Journal of the International Hemp Association, 1995, vol. 2, no. 1, pp. 1417.

[6] I. Bocsa and M. Karus, "Hemp cultivation" in: The cultivation of hemp: botany, varieties, cultivation and harvesting. Sebastopol, USA, 1998, $186 \mathrm{p}$.

[7] L. Grabovska and W. Koziara, "The effect of nitrogen dose, sowing density and time of harvest on development and yields of hemp cultivar Bialobrzeskie", Journal of Natural Fibres, 2005, vol. 2 (4), pp.1-17.

[8] Poradnik plantatora lnu i konopi (koodinator Szalkowski Z.), Poznan, 1994, p.51-61.

[9] H. Burczyk, L. Grabowska, M. Strybe and W. Konczewicz, "Effect of sowing density and date of harvest on yields of industrial hemp". Journal of Natural Fibers, 2009, vol. 6, pp. 204-218.

[10] P. C. Struik, S. Amaducci, M. J. Bullard, N. C. Stutterheim, G. Venturi and H. T. H. Cromack, "Agronomy of fibre hemp (Cannabis sativa L.) in Europe", Industrial Crops and Products, 2000, vol.11, pp. 107-118,.
[11] V. V. Buivydaitè, M. Vaičys, J. Juodis and A. Motuzas, Lietuvos dirvožemiu klasifikacija. Vilnius, 2001, p. 76.

[12] В.П., Понажев и О.В. Медведева, "Методы создания семян льна-долгунца с использованием отбора растений по признакам «мыклость» и «сбежистость»", Инновационные разработки - льноводству: Селекция, семеноводство, возделывание, первичная обработка, экономика. Тверь: Твер.гос.ун-т., 2011. с. 36.

[13] Методики технологической оценки льна и конопли. Moscow, 1961, $182 \mathrm{p}$

[14] P. Tarakanovas and S. Raudonius, Agronominių tyrimu duomenu statistinè analizè taikant kompiuterines programas ANOVA, STAT, SPLIT-PLOT iš paketo SELEKCIJA ir IRRISTAT. Akademija, Kauno r. 58 p., 2003.

[15] Т.А. Рожмина, А.И. Рыжов, Т.А. Кудряшова и Л.М. Голубева, "Генетическое разнообразие мировой коллекции льна-долгунца по параметрам качества льноволокна (Genetic diversity of flax global collection according to fibre quality parameters)”. Инновационные разработки - льноводству: Селекция, семеноводство, возделывание, первичная обработка, экономика. Тверь: Твер.гос.ун-т., 2011, с. 16-17.

[16] Z. Jankauskienè, E. Gruzdevienè, S. Ivanovs and E. Maumevičius, "Investigation of hemp (Cannabis sativa L.) morphological parameters as influenced by seed rate and genotype". Engineering for rural development. 15th International Scientific Conference, May 25-27, 2016, Jelgava, Latvia. Proceedings, Vol. 15, p. 893-897.

[17] Z. Jankauskienė and E. Gruzdevienè, "Genetic recourses: Evaluation of the value of some old Latvian flax accessions", Environment. Technology. Resources: proceedings of the 8th International Scientific and Practical Conference, Rezekne, June 20-22, Vol. II. 2011. Rezekne, 2011, pp. 297-304. 